\title{
MATHEMATICAL MODELLING OF CHEMICAL AGENT REMOVAL BY REACTION WITH AN IMMISCIBLE CLEANSER
}

\author{
M. P. DALWADI* D. O’KIELY ${ }^{\dagger}$, S. J. THOMSON ${ }^{\dagger}$, T. S. KHALEQUE ${ }^{\ddagger}$, AND C. L. HALL ${ }^{\S}$
}

Abstract. When a hazardous chemical agent has soaked into a porous medium, such as concrete, it can be difficult to neutralise. One removal method is chemical decontamination, where a cleanser is applied to react with and neutralise the agent, forming less harmful reaction products. There are often several cleansers that could be used to neutralise the same agent, so it is important to identify the cleanser features associated with fast and effective decontamination. As many cleansers are aqueous solutions while many agents are immiscible with water, the decontamination reaction often takes place at the interface between two phases. In this paper, we develop and analyse a mathematical model of a decontamination reaction between a neat agent and an immiscible cleanser solution. We assume that the reaction product is soluble in both the cleanser phase and the agent phase. At the moving boundary between the two phases, we obtain coupling conditions from mass conservation arguments and the oil-water partition coefficient of the product. We analyse our model using both asymptotic and numerical methods, and investigate how different features of a cleanser affect the time taken to remove the agent. Our results reveal the existence of two regimes characterised by different rate-limiting transport processes, and we identify the key parameters that control the removal time in each regime. In particular, we find that the oil-water partition coefficient of the reaction product is significantly more important in determining the removal time than the effective reaction rate.

Key words. decontamination, surface reaction, moving boundary problem, Stefan problem, asymptotic analysis

AMS subject classifications. 80A32, 92E20, 35C20, 80A22

\section{Introduction.}

1.1. Decontamination in porous media. Chemical spills can be both environmentally and financially disastrous, and a clear understanding of the effectiveness of different clean-up methods is vital for quick and efficient decontamination. Chemical spills are typically neutralised by applying a cleanser solution to the spill which reacts with the contaminating agent to produce less harmful products. With a small number of exceptions, the cleansers used for decontamination are applied as aqueous solutions $[17,19]$. However, many agents of concern are organic compounds with low solubility in water. This means that achieving good mixing of the aqueous decontaminant with the organic agent is often a critical rate-limiting step, and the speed of decontamination is greatly affected by the water-solubility of the contaminating agent $[1,9,10,17,21]$.

The challenges of achieving good mixing of cleanser and agent are particularly pronounced when the agent has soaked into a porous material, such as concrete. In this case, the cleanser and agent cannot be mixed mechanically (e.g. by stirring), and the speed of decontamination is likely to be limited by cleanser and agent transport. Studying the decontamination of porous materials also presents experimental challenges. While some methods have been developed for investigating the behaviour of

\footnotetext{
* Synthetic Biology Research Centre, University of Nottingham, University Park, Nottingham, NG7 2RD, UK (mohit.dalwadi@nottingham.ac.uk)

${ }^{\dagger}$ Mathematical Institute, University of Oxford, Oxford, UK (okiely@maths.ox.ac.uk, thomson@maths.ox.ac.uk)

‡Department of Applied Mathematics, University of Dhaka, Dhaka, Bangladesh (tania.khaleque@du.ac.bd)

$\S$ Mathematics Applications Consortium with Science and Industry, University of Limerick, Castletroy, Limerick, Ireland (cameron.hall@ul.ie)
} 
agents in porous media [20], it is extremely difficult to track the progress of a neutralisation reaction in situ and thus obtain reliable data about the effectiveness of a decontamination protocol [16].

In many cases, multiple different cleanser solutions could be used to neutralise the same agent; for a detailed description of cleanser solutions in current use, and different decontamination reactions and their products, see [19] and [14], respectively. However, only limited data are available about how quickly and how completely a contaminating agent in a porous medium is neutralised by a given cleanser.

Mathematical modelling of decontamination in porous media can give valuable insights into the effectiveness of cleanser solutions by analysing how different physical and chemical properties of agents and cleansers affect the speed and effectiveness of decontamination. This information can be used both to guide the choice of cleanser for a specific application and to inform the development of new cleansers.

1.2. Reactions at phase boundaries. In a general setting, the evolving distributions of agent, cleanser, and reaction products in a porous medium are controlled by (i) the transport of chemical species by diffusion and advection, and (ii) the reactions that occur. If the agent and the cleanser solution are completely immiscible, these reactions only occur at phase boundaries. Reactive transport of chemicals in multiphase systems is important in hydrology and geology, and various mathematical models have been developed to describe reactive transport [4, 5, 12, 13, 15, 18].

In many of these models, the reacting species are in different phases and reactions occur only at phase boundaries. To the best of our knowledge, apart from a study group report on preliminary work [6], the published literature deals only with reactions of this type where one of the reacting species is in a solid phase, within which diffusion and advection can be neglected. In contrast, we are concerned with the reaction between a water-phase cleanser solution and an oil-phase agent, where the important reacting species are in two distinct fluid phases and chemical transport in each phase occurs due to diffusion. In this context, simultaneous transport of the reactive species to the phase boundary is crucial.

As we describe below, our model of decontamination involves mass exchange between two phases (an oil phase and a water phase) at a free boundary. Mass exchange at phase boundaries has been extensively studied in the context of the Stefan problem, a famous model of melting and freezing (see, for example, $[2,3,7$, 8]). While there are important differences between decontamination and the classical Stefan problem, we show that our model reduces to a Stefan problem with kinetic undercooling in certain limits.

1.3. Outline of paper. This paper describes the development and analysis of a model of decontamination in two immiscible phases, where chemical transport is due to diffusion in each phase and the decontamination reaction occurs at the boundary between the two immiscible fluid phases. While real decontamination systems can be very complicated, often involving multiple reactions with multiple products [14], we concentrate on an idealised scenario involving a bimolecular reaction between a neat agent and a cleanser solution to produce a single reaction product. The agent and cleanser solution are assumed to be immiscible, but the neutralisation reaction is assumed to yield a reaction product that gets distributed between the two phases according to a known partition coefficient.

The assumptions that we make in developing our model allow us to analyse how the salient features of the decontamination system (e.g. the reaction rate constant, the diffusion constants of different species, the partition coefficient for the distribution 
of reaction product between phases) affect the overall speed of decontamination. As discussed above, since we can find no references in the present literature that discuss (let alone quantify) how such features affect decontamination speed, this paper represents a valuable first step in understanding the processes that control the effectiveness of decontamination.

In $\S 2$, we describe the development and nondimensionalisation of our model, paying particular attention to the boundary conditions that hold at the moving interface. This leads to equations (12)-(15), which give a dimensionless representation of our model, transformed to fixed spatial domains. In $\S 3$ we consider the early-time asymptotic behaviour of the model and present numerical solutions to the system. While not important for determining the overall speed of decontamination, the early-time analysis is essential for developing accurate and efficient numerical schemes for solving (12)-(15). We also introduce two measures of agent removal time that can be used to characterise decontamination.

We follow this in $\S 4$ with an asymptotic analysis of the governing equations for long time. Under the assumption that the initial agent layer is deep, we find that the long-time dynamics of the model fall into one of two regimes. We investigate the behaviour of the system in both of these regimes, providing asymptotic results where possible. These results allow us to gain deeper physical insight into the underlying system and predict the most important parameters for decontamination. We discuss the physical implications of our work in $\S 5$, where we present our results in dimensional form and thus identify some desirable features of cleansers.

\section{Model development.}

2.1. Model outline. Throughout this paper, we use SI units to indicate the dimensions of parameters and variables when they are first introduced. We consider a one-dimensional porous medium of length $\bar{L}[\mathrm{~m}]$, with the $\bar{x}$-axis pointing into the medium, as shown in figure 1. Before the decontamination reaction begins, the neat agent has penetrated to the end of the porous medium, so that the agent entirely occupies the region $0<\bar{x}<\bar{L}$. Then, at time $\bar{t}=0$, an aqueous solution of cleanser with uniform concentration $\bar{c}_{0}\left[\mathrm{~mol} \mathrm{~m}^{-3}\right]$ is introduced to the surface at $\bar{x}=0$. The aqueous phase (containing the cleanser) and the oil phase (containing the agent) are assumed to be immiscible, but the position of the interface between them, $\bar{s}(\bar{t})[\mathrm{m}]$, can change in time. We assume that the porous medium is homogeneous and fully saturated with fluid. As a result of this, the porosity of the medium does not play an explicit role in our analysis.

At the interface between the phases, the cleanser and the agent react irreversibly. This reaction consumes cleanser and agent and leads to the formation of a neutral product that is soluble in both the aqueous phase and the oil phase. In the aqueous phase, the evolving concentration of cleanser is given by $\bar{c}(\bar{x}, \bar{t})\left[\mathrm{mol} \mathrm{m}^{-3}\right]$ and the evolving concentration of reaction product is given by $\bar{p}(\bar{x}, \bar{t})\left[\mathrm{mol} \mathrm{m}^{-3}\right]$.

In the oil phase, we assume that the product and agent form an ideal mixture whose molar volume is independent of composition. We describe the evolving composition of the oil phase using the volume fraction of reaction product in the oil phase, $\bar{\phi}(\bar{x}, \bar{t})$ [dimensionless]. Since the oil phase contains only agent and product, the volume fraction of contaminant in the oil phase is $1-\bar{\phi}$. Ideality of the agent-product mixture implies that the diffusion of product in agent is equivalent to the diffusion of agent in product, and hence we can represent diffusive transport in the oil phase using a single diffusion equation for $\bar{\phi}$.

We assume that all transport of cleanser, agent, and product within their re- 


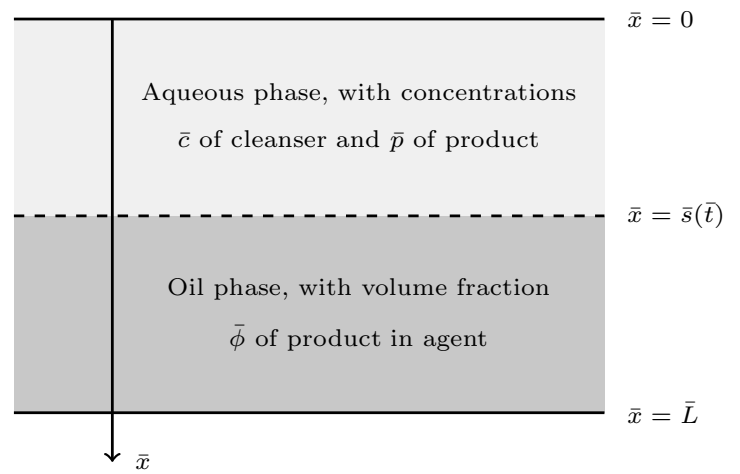

FIG. 1. Schematic diagram of the physical problem. Over time, the interface between the aqueous phase and the oil phase moves down (in the direction of increasing $\bar{x}$ ) as agent is consumed.

spective phases is due to diffusion. Assuming Fickian diffusion and exploiting the assumption that the porous medium is saturated and uniformly porous, this gives the governing equations for the evolution of $\bar{c}, \bar{p}$ and $\bar{\phi}$ to be

$$
\begin{aligned}
\bar{c}_{\bar{t}} & =\bar{D}_{c} \bar{c}_{\bar{x} \bar{x}}, \\
\bar{p}_{\bar{t}} & =\bar{D}_{p} \bar{p}_{\bar{x} \bar{x}}, \\
\bar{\phi}_{\bar{t}} & =\bar{D}_{\phi} \bar{\phi}_{\bar{x} \bar{x}},
\end{aligned}
$$

$$
\begin{aligned}
& \text { for } 0<\bar{x}<\bar{s}(\bar{t}) \text { and } \bar{t}>0, \\
& \text { for } 0<\bar{x}<\bar{s}(\bar{t}) \text { and } \bar{t}>0, \\
& \text { for } \bar{s}(\bar{t})<\bar{x}<\bar{L} \text { and } \bar{t}>0,
\end{aligned}
$$

where $\bar{D}_{c}\left[\mathrm{~m}^{2} \mathrm{~s}^{-1}\right], \bar{D}_{p}\left[\mathrm{~m}^{2} \mathrm{~s}^{-1}\right]$ and $\bar{D}_{\phi}\left[\mathrm{m}^{2} \mathrm{~s}^{-1}\right]$ are the effective diffusion coefficients within a porous medium of the cleanser in aqueous solution, the product in aqueous solution, and the agent/product in the oil phase respectively. Subscripts of $\bar{t}$ or $\bar{x}$ denote partial derivatives with respect to time and space respectively.

At $\bar{x}=0$, we assume that the cleanser is continually being replenished at a constant concentration $\bar{c}_{0}\left[\mathrm{~mol} \mathrm{~m}^{-3}\right]$ while the reaction product is continually being removed from the system. At $\bar{x}=\bar{L}$, we assume that there is a fixed boundary that no species can pass through. This yields the boundary conditions

$$
\begin{gathered}
\bar{c}=\bar{c}_{0}, \quad \bar{p}=0 \quad \text { for } \bar{x}=0, \\
\bar{\phi}_{\bar{x}}=0 \quad \text { for } \bar{x}=\bar{L} .
\end{gathered}
$$

At $\bar{t}=0$, the interface between the phases is located at $\bar{x}=0$, and there is no oily product yet, so the initial conditions are

$$
\bar{\phi}=0, \quad \bar{s}=0 \quad \text { for } \bar{t}=0 .
$$

2.2. Interfacial conditions. We assume that the agent and the cleanser react in an irreversible bimolecular reaction at the phase boundary to produce the reaction product. For simplicity, we assume that the rate of the decontamination reaction is proportional to the bulk concentrations of the two reagents in the neighbourhood of the phase boundary. While this approach means that we neglect the kinetics of absorption and desorption, Kumar et al. [11] have shown that it is consistent with more complicated kinetic schemes in appropriate limits.

Mathematically, we describe the kinetics of decontamination by introducing the total molar flux of reaction, $\bar{R}\left[\mathrm{~mol} \mathrm{~m}^{-2} \mathrm{~s}^{-1}\right]$. This represents the consumption rate of cleanser and agent (and, equivalently, the production rate of reaction product) per 
unit area of the interface. Using our assumption that $\bar{R}$ is proportional to the amounts of cleanser and agent available at the oil-water interface, we obtain

$$
\bar{R}=\bar{k} \bar{c}[\bar{s}(\bar{t}), \bar{t}]\{1-\bar{\phi}[\bar{s}(\bar{t}), \bar{t}]\},
$$

where $\bar{k}\left[\mathrm{~m} \mathrm{~s}^{-1}\right]$ is a constant of proportionality which we refer to as the effective rate constant.

We use (5) to obtain interfacial conditions on $\bar{c}, \bar{\phi}$, and $\bar{p}$, noting that the total amounts of cleanser, agent, and product in the system (in moles) are given by

$$
\begin{aligned}
\mathcal{C}(\bar{t}) & =\bar{A} \int_{0}^{\bar{s}(\bar{t})} \bar{c}(\bar{x}, \bar{t}) \mathrm{d} \bar{x}, \\
\mathcal{A}(\bar{t}) & =\frac{\bar{A}}{\bar{V}_{m}} \int_{\bar{s}(\bar{t})}^{\bar{L}} 1-\bar{\phi}(\bar{x}, \bar{t}) \mathrm{d} \bar{x}, \\
\mathcal{P}(\bar{t}) & =\bar{A} \int_{0}^{\bar{s}(\bar{t})} \bar{p}(\bar{x}, \bar{t}) \mathrm{d} \bar{x}+\frac{\bar{A}}{\bar{V}_{m}} \int_{\bar{s}(\bar{t})}^{\bar{L}} \bar{\phi}(\bar{x}, \bar{t}) \mathrm{d} \bar{x},
\end{aligned}
$$

where $\bar{A}\left[\mathrm{~m}^{2}\right]$ is the area of the spill, and $\bar{V}_{m}\left[\mathrm{~m}^{3} \mathrm{~mol}^{-1}\right]$ is the molar volume of the agent/product mixture. Since we have assumed that the agent and product form an ideal mixture, $\bar{V}_{m}$ is a constant independent of $\bar{\phi}$.

Differentiating (6a) using Leibniz's rule, we recognise that the molar flux of cleanser into the oil-water interface is given by $\bar{D}_{c} \bar{c}_{\bar{x}}+\bar{c} \bar{s}_{\bar{t}}$, evaluated at $\bar{x}=\bar{s}(\bar{t})$. Since the removal of cleanser at the oil-water interface happens via the decontamination reaction, we use (5) to obtain

$$
\bar{D}_{c} \bar{c}_{\bar{x}}+\bar{c} \bar{s}_{\bar{t}}=-\bar{k} \bar{c}(1-\bar{\phi}) \quad \text { on } \bar{x}=\bar{s}(\bar{t}) \text { for } \bar{t}>0 .
$$

Repeating this process with equations (6b) and (6c), we obtain two further interfacial conditions,

$$
\begin{gathered}
-\frac{\bar{D}_{\phi}}{\bar{V}_{m}} \bar{\phi}_{\bar{x}}+\frac{1-\bar{\phi}}{\bar{V}_{m}} \bar{s}_{\bar{t}}=\bar{k} \bar{c}(1-\bar{\phi}) \quad \text { on } \bar{x}=\bar{s}(\bar{t}) \text { for } \bar{t}>0, \\
\bar{D}_{p} \bar{p}_{\bar{x}}+\bar{p} \bar{s}_{\bar{t}}=\frac{1}{\bar{V}_{m}} \bar{s}_{\bar{t}} \quad \text { on } \bar{x}=\bar{s}(\bar{t}) \text { for } \bar{t}>0,
\end{gathered}
$$

where $(7 \mathrm{c})$ has been rearranged using $(7 \mathrm{~b})$.

We obtain the final interfacial condition by assuming that the reaction product is locally in equilibrium between the oil phase and the water phase. Thus, the concentrations of product on either side of the interface are related via a partition constant, and the final interfacial condition is

$$
\frac{\bar{\phi}}{\bar{V}_{m}}=\mathcal{K} \bar{p} \quad \text { on } \bar{x}=\bar{s}(\bar{t}) \text { for } \bar{t}>0,
$$

where $\mathcal{K}$ [dimensionless] is the oil-water partition constant of the reaction product. In practice, $\mathcal{K}$ can be estimated from octanol-water partition constants, which have been measured for a range of relevant compounds [14].

We note that the interfacial conditions stated in (7) are similar to those considered in Stefan problems with kinetic undercooling. To see this, consider the limit $\mathcal{K} \rightarrow 0$, where no reaction product enters the oil phase. In this case, the interfacial conditions for $\bar{c}$ and $\bar{s}$ can be reduced to

$$
\bar{D}_{c} \bar{c}_{\bar{x}}=-\bar{k} \bar{c}-\bar{c} \bar{s}_{\bar{t}}
$$




$$
\bar{s}_{\bar{t}}=\bar{k} \bar{V}_{m} \bar{c},
$$

which are equivalent to the classic Stefan problem with kinetic undercooling [8].

2.3. Nondimensionalisation and transformation to a fixed domain. As part of our nondimensionalisation, we pre-empt the challenges associated with numerical solution on a domain with a moving boundary, and introduce a boundaryfixing transformation by defining 'upper' and 'lower' spatial variables, $\xi \in[0,1]$ and $\eta \in[0,1]$, respectively, so that

$$
\xi:=\frac{\bar{x}}{\bar{s}(\bar{t})}, \quad \eta:=\frac{\bar{L}-\bar{x}}{\bar{L}-\bar{s}(\bar{t})} .
$$

With these definitions, the boundary conditions applied at $\bar{x}=0$ and $\bar{x}=\bar{L}$ are now applied at $\xi=0$ and $\eta=0$, respectively, while the interfacial conditions applied at the moving boundary $\bar{x}=\bar{s}(\bar{t})$ are now applied at the fixed boundaries $\xi=1$ and $\eta=1$.

We nondimensionalise our dependent variables by introducing

$$
\bar{c}(\bar{x}, \bar{t}):=\frac{c(\xi, t)}{\bar{V}_{m}}, \quad \bar{p}(\bar{x}, \bar{t}):=\frac{p(\xi, t)}{\bar{V}_{m}}, \quad \bar{s}(\bar{t}):=\frac{\bar{D}_{c}}{\bar{k}} s(t), \quad \bar{t}:=\frac{\bar{D}_{c}}{\bar{k}^{2}} t,
$$

and, observing that $\bar{\phi}$ is already dimensionless, we also use $\bar{\phi}(\bar{x}, \bar{t})=\phi(\eta, t)$.

Applying the nondimensionalisation and the boundary-fixing transformation described above, we identify the following set of five dimensionless parameters that prescribe the system:

$$
\beta:=\bar{c}_{0} \bar{V}_{m}, \quad D_{p}:=\frac{\bar{D}_{p}}{\bar{D}_{c}}, \quad D_{\phi}:=\frac{\bar{D}_{\phi}}{\bar{D}_{c}}, \quad \mathcal{K}, \quad d:=\frac{\bar{L} \bar{k}}{\bar{D}_{c}} .
$$

We discuss the decontamination of sulfur mustard in $\S 5.3$, for which we obtain typical parameter values of $\beta \approx 0.03-8$ and $\mathcal{K} \approx 0.14-7.1$. However, diffusion and reaction coefficients are more difficult to obtain. In $\S 4$, we explore in detail the case where $d$ is much larger than the other parameters in the system, modelling deep spills of agent.

In rescaled form, the governing equations (1) become

$$
\begin{aligned}
c_{\xi \xi}+\dot{s} s \xi c_{\xi} & =s^{2} c_{t} & & \text { for } 0<\xi<1 \text { and } t>0, \\
D_{p} p_{\xi \xi}+\dot{s} s \xi p_{\xi} & =s^{2} p_{t} & & \text { for } 0<\xi<1 \text { and } t>0, \\
D_{\phi} \phi_{\eta \eta}-\dot{s}(d-s) \eta \phi_{\eta} & =(d-s)^{2} \phi_{t} & & \text { for } 0<\eta<1 \text { and } t>0,
\end{aligned}
$$

where $\dot{s}=\mathrm{d} s / \mathrm{d} t$ and subscripts of $\xi, \eta$, and $t$ represent partial differentiation. We observe that $s(t) \in[0, d]$, and hence $(d-s)$ is always nonnegative.

The initial conditions (4) become

$$
\phi(\eta, 0)=0, \quad s(0)=0,
$$

while the boundary conditions (2) and (3) become

$$
c(0, t)=\beta, \quad p(0, t)=0, \quad \phi_{\eta}(0, t)=0 .
$$

Additionally, the interfacial conditions (7) are now

$$
c_{\xi}+s c=s c(\phi-\dot{s}),
$$




$$
\begin{aligned}
D_{\phi} \phi_{\eta} & =-(d-s)(\dot{s}-c)(1-\phi), \\
D_{p} p_{\xi} & =s \dot{s}(1-p), \\
\phi & =\mathcal{K} p,
\end{aligned}
$$

where all variables are evaluated for $t>0$ and at $\xi=1$ or $\eta=1$, as appropriate. The full dimensionless system is then described by (12)-(15).

\section{Early-time asymptotics and numerical solutions.}

3.1. Early-time asymptotics. Since the aqueous phase is initially absent from the system, the boundary-fixing transformation described in $\S 2.3$ is singular at $t=0$. We circumvent the numerical difficulties created by this singularity by calculating the early-time behaviour of the system as $t \rightarrow 0^{+}$. We use these results to start our computations at a small but finite time.

We begin by using (12)-(15) to obtain consistent initial conditions for $c$ and $p$. For small $s$, assuming that all other terms are bounded, (12) yields $c_{\xi \xi}=p_{\xi \xi}=0$ and (15) yields $c_{\xi}(1,0)=p_{\xi}(1,0)=0$. Applying the boundary conditions (14), we obtain

$$
c(\xi, 0)=\beta, \quad p(\xi, 0)=0 .
$$

We can now obtain early-time results. Rescaling $t$ with an arbitrary small parameter and seeking asymptotic balances in (12) and (15) where $c, p, \phi$ and $s$ are close to their initial values, we find that $s=O(t), c=\beta+O(t), \phi=O(t)$, and $p=O(t)$. Solving for $c, p$, and $s$ leads to the explicit results

$$
c \sim \beta-\beta^{2}(1+\beta) \xi t, \quad p \sim \frac{\beta^{2} \xi t}{D_{p}}, \quad s \sim \beta t, \quad \text { as } t \rightarrow 0^{+} .
$$

In order to obtain explicit early-time results for $\phi$, we make the assumption that $d$ is large. This corresponds to a deep spill of chemical agent, and is the main focus of our analysis in $\S 4$. In this case we seek a boundary layer solution for $\phi$ near $\eta=1$; we introduce the rescaled spatial variable $X=d(1-\eta)$ to obtain the leading-order early-time system

$$
\begin{array}{r}
D_{\phi} \phi_{X X}+\beta \phi_{X}=\phi_{t} \quad \text { for } X>0 \text { and } t>0, \\
\phi=\frac{\beta \mathcal{K} t}{D_{p}} \quad \text { on } X=0 \text { for } t>0, \\
\phi \rightarrow 0 \quad \text { as } X \rightarrow \infty \text { for } t>0, \\
\phi=0 \quad \text { on } t=0 \text { for } X>0 .
\end{array}
$$

This is solved by

$$
\phi=\frac{\beta \mathcal{K}}{2 D_{p}}\left\{(\beta t+X) \operatorname{erfc}\left(\frac{X+\beta t}{2 \sqrt{D_{\phi} t}}\right)+e^{-\beta X / D_{\phi}}(\beta t-X) \operatorname{erfc}\left(\frac{X-\beta t}{2 \sqrt{D_{\phi} t}}\right)\right\},
$$

where $\operatorname{erfc}(z)$ is the complementary error function.

3.2. Numerical solutions. The full dimensionless problem (12)-(15) is solved using the method of lines. We use a uniform mesh for $\xi$ and, to resolve the boundary layer observed in the previous section, we use a non-uniform mesh with logarithmically spaced points for $\eta$. The logarithmic spacing is focused near $\eta=1$ and is only used 

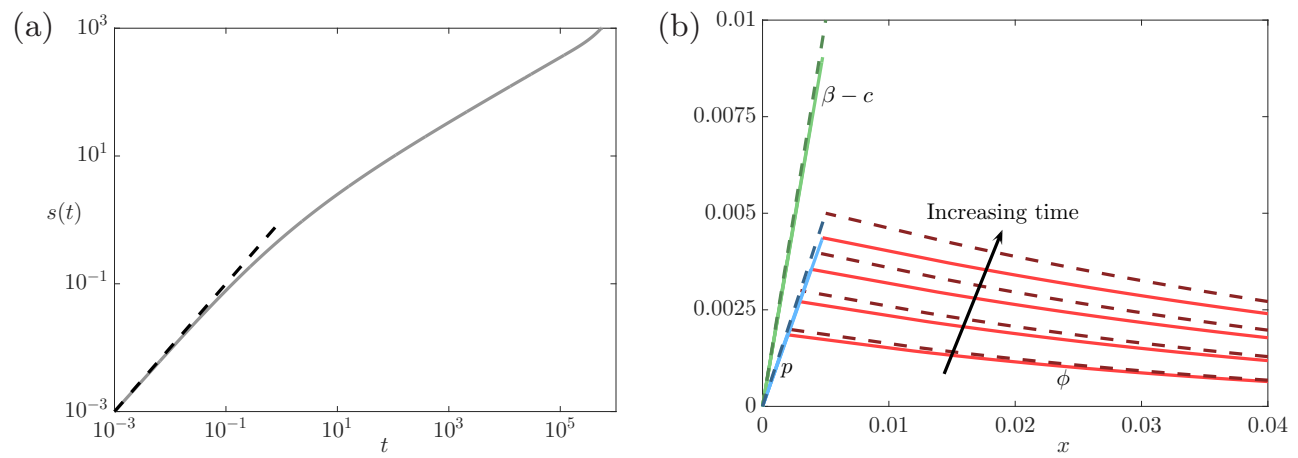

FIG. 2. (Colour online) Comparison of numerical solutions (solid) and asymptotic results (dashed) at early times for $\beta=\mathcal{K}=D_{\phi}=D_{p}=1, d=10^{3}$. (a) The position of the moving boundary. (b) The change from the initial conditions at early time. The decrease in cleanser concentration profile, $\beta-c$ (green), increase in product concentration in aqueous phase, $p$ (blue), and increase in product concentration in oily phase, $\phi\left(\right.$ red), at times $t=2 \times 10^{-3}, 3 \times 10^{-3}, 4 \times$ $10^{-3}, 5 \times 10^{-3}$ with the arrow denoting an increase in time. The numerical solutions start from $t=10^{-3}$ as described in the text.

while $s(t)<d / 4$; after this point in time, we use a uniform mesh. We discretise (12)(15) in space using second-order finite differences, and integrate in time with ode15s in MATLAB, using the early-time solutions (17) and (19) to provide consistent initial conditions. We use 'ghost' points just outside the domain to impose the boundary conditions, using (15c) for $\dot{s},(15 \mathrm{a})$ for $c$, (15d) for $p$, and (15b) for $\phi$ at the free boundary. We verify the early-time solution by comparing full numerical solutions initiated at $t=10^{-3}$ with the early-time asymptotic solutions (17) and (19) in figure 2, and we observe good agreement. The asymptotic predictions (17) for $\beta-c$ and $p$ do not change in time when rescaled for the physical domain via (9), and the early-time numerical solutions exhibit the same behaviour.

In figures 3 and 4 we show the evolution of the cleanser-agent-product system for illustrative parameter values, namely $\beta=D_{\phi}=D_{p}=1, d=10^{3}$, with $\mathcal{K}=10$ in figure 3 and $\mathcal{K}=1$ in figure 4 . As we discuss further in $\S 5$, values of $\mathcal{K}$ within an order of magnitude of unity are realistic. Additionally, we expect all diffusion constants to be comparable. The choices of $\mathcal{K}$ that we make in figures 3 and 4 enable us to demonstrate how different parameter choices lead to qualitatively different solution behaviours.

In both figure 3 and 4 , we observe that the interface moves in the positive $x$ direction, consuming the agent. The interface reaches the lower boundary almost an order of magnitude faster for $\mathcal{K}=1$ than for $\mathcal{K}=10$. Additionally, we observe that the concentration profiles of cleanser, $c$, and product in the upper and lower regions, $p$ and $\phi$, respectively, are sensitive to the partition coefficient $\mathcal{K}$. For $\mathcal{K}=10$, we observe that $\phi$ reaches values close to 1 (so agent concentration is close to 0 ) while the interface is still far from the lower boundary (figure 3) whereas, for $\mathcal{K}=1, \phi<1$ throughout the reaction and $c$ appears to vanish close to the interface (figure 4). There is a significant difference in the system behaviour between these two cases, and we can see this more clearly by considering the proportion of remaining agent in the system, defined by

$$
\Phi(t)=\frac{d-s(t)}{d} \int_{0}^{1}(1-\phi(\eta, t)) \mathrm{d} \eta
$$



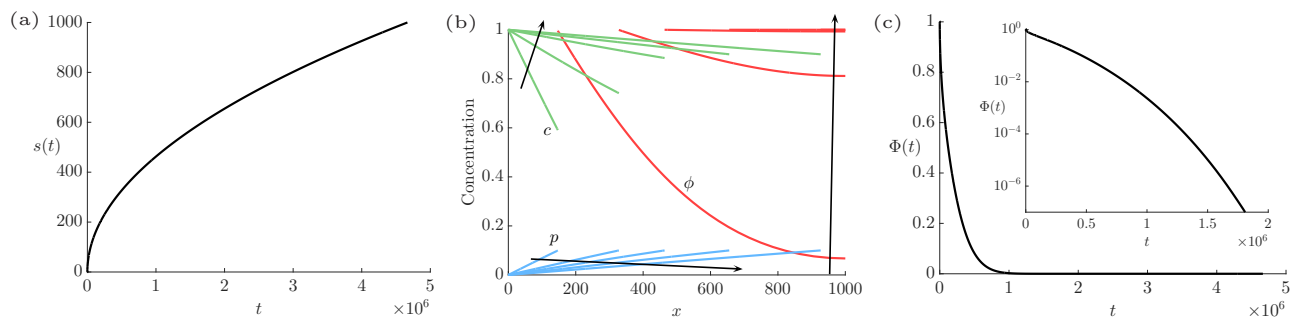

FIG. 3. (Colour online) Numerical results for the system (12)-(15) using the parameter values $\beta=D_{\phi}=D_{p}=1, \mathcal{K}=10$, and $d=10^{3}$. (a) The position of the moving boundary, $s(t)$. (b) The concentrations of the cleanser, $c$ (green), product in aqueous phase, $p$ (blue), and product in oily phase, $\phi$ (red), at (non-uniform) times $t=1 \times 10^{5}, 5 \times 10^{5}, 1 \times 10^{6}, 2 \times 10^{6}, 4 \times 10^{6}$, where the arrows denote increasing time. (c) The proportion of remaining contaminant in the system, $\Phi(t)$, defined in (20a). The inset shows a log-lin version of the same function.
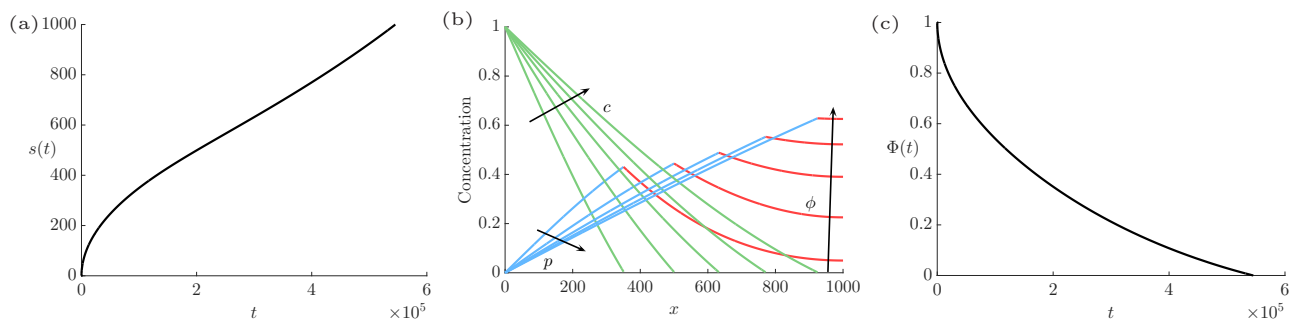

FIG. 4. (Colour online) Numerical results for the system (12)-(15) using the parameter values $\beta=D_{\phi}=D_{p}=\mathcal{K}=1$, and $d=10^{3}$. (a) The position of the moving boundary, $s(t)$. (b) The concentrations of the cleanser, $c$ (green), product in aqueous phase, $p$ (blue), and product in oily phase, $\phi$ (red), at times $t=1 \times 10^{5}, 2 \times 10^{5}, 3 \times 10^{5}, 4 \times 10^{5}, 5 \times 10^{5}$, where the arrows denote increasing time. (c) The proportion of remaining contaminant in the system, $\Phi(t)$, defined in (20a).

For $\mathcal{K}=10$, we see that most of the agent is consumed significantly before the moving interface reaches the lower boundary (figure 3c) and we see that, for example, $\Phi<10^{-4}$ before the interface has reached a third of the way to the lower boundary. However, for $\mathcal{K}=1$ the agent appears to be consumed more uniformly as the interface moves, and the remaining agent in the system is only small when the interface is close to the lower boundary (figure 4c).

In order to make quantitative comparisons of different decontamination simulations, we now introduce two different measures of the time taken to decontaminate the system. The first of these is a measure of the time until complete agent removal, $t_{f}$; we refer to this as the final time and define it by

$$
t_{f}:=\min \{t>0: s(t)=d\}
$$

While $t_{f}$ is the time taken for the interface to reach the lower boundary (and hence the time taken to completely remove all agent), it is possible that most of the agent reacts with the cleanser long before $t=t_{f}$, as illustrated in figure 3 . To investigate this scenario, we introduce a second measure of removal time, $t_{e}$. We refer to $t_{e}$ as the effective removal time, and it corresponds to the time taken until the total amount of remaining agent drops below some safe threshold. We define $t_{e}$ by

$$
t_{e}:=\min \{t>0: \Phi(t)<\varepsilon\},
$$




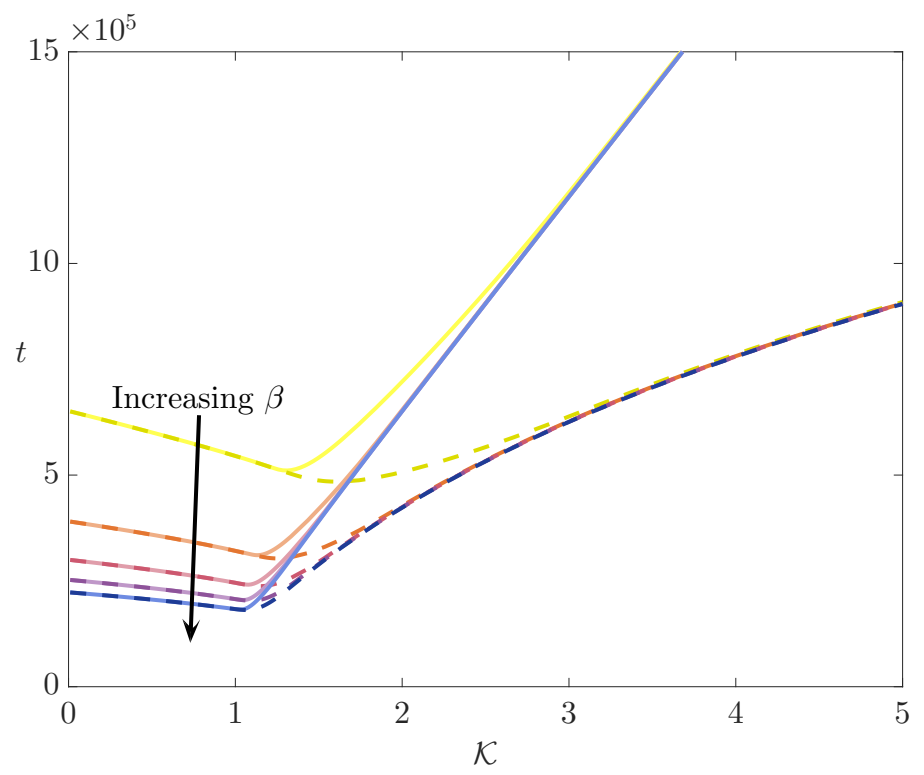

FIG. 5. (Colour online) The final time $t_{f}$ (solid lines) and effective removal time $t_{e}$ (dashed) as functions of $\mathcal{K}$, calculated using numerical solutions of (12)-(15). Each line denotes a different value of $\beta$, corresponding to $\beta=1,2,3,4$, and 5 . We use parameter values $D_{\phi}=D_{p}=1$, and $d=10^{3}$.

where $\varepsilon$ represents the proportion of the original agent that remains when the safe threshold is reached. We take $\varepsilon=10^{-4}$ throughout this paper. The fact that $t_{e}<t_{f}$ follows from the definitions in (20), from which we also expect the final and effective removal times to be close to one another, except for in scenarios where $\phi \approx 1$ before the interface reaches the lower boundary.

As $\mathcal{K}$ increases, for a given $\beta$, we observe that the final times and the effective removal times are close to one another until $\mathcal{K}$ reaches some threshold value, after which the two measures of removal time diverge (figure 5). Moreover, we observe that neither measure of removal time is a monotone function of $\mathcal{K}$; instead, there are optimal $\mathcal{K}$ values at which the final time or effective removal time are minimised, and these optimal values of $\mathcal{K}$ depend weakly on $\beta$. Additionally, we observe that both measures of removal time depend strongly on $\beta$ when $\mathcal{K}$ is small, but are effectively independent of $\beta$ when $\mathcal{K}$ is large.

To further understand the dependence of $t_{f}$ and $t_{e}$ on $\beta, \mathcal{K}$, and the other model parameters, we proceed by analysing (12)-(15) using asymptotic methods in the physically relevant limit of large $d$. This will enable us to make general deductions about the system and will provide physical insight into the parameters that control the decontamination process. Additionally, we investigate why the final and effective removal times diverge in certain parameter regimes.

\section{Long-time asymptotic analysis.}

4.1. Large $d$ assumption. We now explore in detail the scenario where there is a deep spill of agent, so that $d \gg 1$. In particular, we investigate the case where $d$ is much larger than the other parameters in the system. Within this limit, we find that different regimes arise for quantifiably different values of the remaining dimensionless parameters. 
As we are interested in the removal times $t_{f}$ and $t_{e}$, defined in (20), we consider the regime where $s=O(d)$. The scalings for this long-time regime are

$$
t=d^{2} T, \quad s=d S,
$$

and hence $S \in[0,1]$. The time scaling explains the magnitude of the $y$-axis in figure 5 , and means that figure 5 will be valid for general large $d$ with a suitable scaling of the $y$-axis. Expanding in inverse powers of $d$ and retaining only leading-order terms, the governing equations (12) become

$$
\begin{aligned}
c_{\xi \xi}+S S_{T} \xi c_{\xi} & =S^{2} c_{T} \quad \text { for } T>0 \text { and } \xi \in(0,1), \\
D_{p} p_{\xi \xi}+S S_{T} \xi p_{\xi} & =S^{2} p_{T} \quad \text { for } T>0 \text { and } \xi \in(0,1), \\
D_{\phi} \phi_{\eta \eta}+S_{T}(S-1) \eta \phi_{\eta} & =(S-1)^{2} \phi_{T} \quad \text { for } T>0 \text { and } \eta \in(0,1) .
\end{aligned}
$$

Similarly, the leading-order boundary conditions from (14) become

$$
c(0, T)=\beta, \quad p(0, T)=0, \quad \phi_{\eta}(0, T)=0 \quad \text { for } T>0,
$$

and the leading-order interfacial conditions from (15) become

$$
\begin{aligned}
(1-\phi) c & =0, \\
\frac{S-1}{S} c_{\xi}-D_{\phi} \phi_{\eta} & =(1-S) S_{T}(c+1-\phi), \\
D_{p} p_{\xi} & =S S_{T}(1-p), \\
\mathcal{K} p & =\phi,
\end{aligned}
$$

where all functions are evaluated for $T>0$ and at $\xi=1$ or $\eta=1$, as appropriate. The full leading-order system for long time is then given by (22)-(24).

We see that (24a) offers two possibilities for the behaviour of this system, where either $\phi=1$ or $c=0$ at the interface. Both scenarios are observed numerically, as illustrated in figures $3 \mathrm{~b}$ and $4 \mathrm{~b}$. These distinct scenarios arise due to different rate-limiting mechanisms which we describe in $\S 4.2$ and $\S 4.3$. Once we have derived asymptotic solutions within each of these regimes, we show that they are associated with different parameter regimes. This analysis is described in $\S 4.4$, where we additionally develop an a priori classification based on the system parameters. From figures 3 and 4 , it appears that $c=0$ is associated with larger $\mathcal{K}$, and $\phi=1$ is associated with smaller $\mathcal{K}$; we formalise this observation in $\S 4.4$.

In the case where $\phi=1$ at the interface, which we refer to as Regime I, agent in the oil phase is consumed as soon as it reaches the interface and hence the rate-limiting step is the removal of oily-phase product from the neighbourhood of the interface, which is in turn controlled by the transport and removal of aqueous product to/at the upper boundary. In this regime, we will also show that the vast majority of agent is removed before the interface reaches the lower boundary, resulting in $t_{e}$ being significantly shorter than $t_{f}$. In the case where $c=0$ on the reaction interface, which we refer to as Regime II, cleanser in the aqueous phase is consumed as soon as it reaches the interface and hence the rate-limiting step is the transport of cleanser to the interface. We now consider Regime I.

4.2. Regime I: Decontamination limited by product removal. In this section, we consider the regime where $\phi=1$ at the oil-water interface. We see from figure 3 that this case can lead to $\phi \approx 1$ throughout the oil phase before the interface 
reaches the lower boundary, and thus can result in an effective removal time, $t_{e}$, that is significantly less than the final time, $t_{f}$. We therefore expect this regime to explain the disparity between $t_{e}$ and $t_{f}$ in figure 5 .

In Regime I, the relevant interfacial conditions from (24) are

$$
\begin{aligned}
\phi & =1, \\
\frac{S-1}{S} c_{\xi}-D_{\phi} \phi_{\eta} & =(1-S) S_{T} c, \\
D_{p} p_{\xi} & =S S_{T}(1-p), \\
p & =1 / \mathcal{K} .
\end{aligned}
$$

The leading-order system in this regime is thus given by (22), (23), and (25).

As the interfacial conditions $(25 \mathrm{c}, \mathrm{d})$ only involve the dependent variables $p$ and $S$, we can solve for these variables independently of $c$ and $\phi$, and hence the system decouples. Moreover, as $p$ now satisfies a Dirichlet condition on the interface, $p$ exhibits similar behaviour to the classic Stefan problem [3, 7]. Solving the system given by (22b), (23b), and (25c,d), we obtain

$$
p(\xi)=\frac{\operatorname{erf} \lambda_{p} \xi}{\mathcal{K} \operatorname{erf} \lambda_{p}}, \quad S(T)=2 \lambda T^{1 / 2} .
$$

Here, $\operatorname{erf}(z)$ is the error function, $\lambda_{p}=\lambda / D_{p}^{1 / 2}$, and $\lambda_{p}$ satisfies the transcendental equation

$$
\mathcal{K}=1+\frac{e^{-\lambda_{p}^{2}}}{\lambda_{p} \sqrt{\pi} \operatorname{erf} \lambda_{p}} .
$$

We note that (27) only has solutions when $\mathcal{K}>1$; we discuss this in more detail in $\S 4.4$. Inserting scaling (26) into definition (20b), we deduce that, in this regime, the final time

$$
\frac{t_{f}}{d^{2}}=\frac{1}{4 \lambda^{2}}+O(1 / d) \quad \text { as } d \rightarrow \infty,
$$

where $\lambda$ is defined in (27). We note that the only dimensionless parameters that affect $t_{f}$ are $\mathcal{K}$ and $D_{p}$, so of the three diffusion processes occurring in the system the diffusion of product in aqueous solution is the most important.

Our task is now to solve for the remaining variables, $c$ and $\phi$. Using (26), the system decouples further, and we can first solve for $\phi$ from (22c), (23c), and (25a), then solve for $c$ from (22a), (23a), and (25b). To solve this reduced system, we must obtain effective initial conditions, and this is carried out in a similar manner to the analysis in $\S 3.1$. That is, we now look for a small-time solution to the reduced problem, and we refer to this as the intermediate-time solution.

As $T \rightarrow 0^{+}$, we make the formal scalings $T=\delta \tilde{T}$ and $\eta=1-\delta^{1 / 2} \tilde{X}$, where $\delta \ll 1$ is an arbitrary small parameter. We look for solutions where $c=c(\xi)$ and $\phi=\phi(\tilde{X}, \tilde{T})$ in the system (22), (23), and (25), essentially seeking the long-time solution to the problem with an infinite lower domain. At leading order in $\delta$, the governing equations $(22 \mathrm{a}, \mathrm{c})$ are

$$
\begin{aligned}
& c_{\xi \xi}+2 \lambda^{2} \xi c_{\xi}=0 \quad \text { for } \xi \in(0,1), \\
& D_{\phi} \phi_{\tilde{X} \tilde{X}}+\frac{\lambda}{\tilde{T}^{1 / 2}} \phi_{\tilde{X}}=\phi_{\tilde{T}} \quad \text { for } \tilde{X}>0 \text { and } \tilde{T}>0
\end{aligned}
$$


the fixed boundary condition (23a) is

$$
c(0)=\beta
$$

and the interfacial conditions $(25 \mathrm{a}, \mathrm{b})$ are

$$
\begin{aligned}
\phi(0, \tilde{T}) & =1 \\
c_{\xi}(1)+2 \lambda^{2} c(1) & =2 \lambda D_{\phi} \tilde{T}^{1 / 2} \phi_{\tilde{X}}(0, \tilde{T}) .
\end{aligned}
$$

Finally, the matching condition for $\phi$ is

$$
\phi \rightarrow 0 \quad \text { as } \tilde{X} \rightarrow \infty \text {. }
$$

The system (29)-(32) is solved by

$$
\begin{aligned}
& c \sim \beta-D_{\phi}\left(\frac{\beta \lambda_{\phi} \operatorname{erfc} \lambda_{\phi}+e^{-\lambda_{\phi}^{2}} / \sqrt{\pi}}{\lambda \operatorname{erf} \lambda+e^{-\lambda^{2}} / \sqrt{\pi}}\right) \frac{\operatorname{erf}(\lambda \xi)}{\operatorname{erfc} \lambda_{\phi}}, \\
& \phi \sim \frac{\operatorname{erfc}\left((1-\eta) / \sqrt{4 D_{\phi} T}+\lambda_{\phi}\right)}{\operatorname{erfc} \lambda_{\phi}}
\end{aligned}
$$

where $\lambda_{\phi}=\lambda / D_{\phi}^{1 / 2}$ and we have re-written the solution for $\phi$ in terms of $\eta$ and $T$. We note that the long-time solutions to the modified problem with a semi-infinite lower domain in Regime I are given by (26) and (33), where $\lambda$ is the solution to (27).

Thus, in Regime I, we have reduced the task of fully understanding our system to numerically solving the system $(22 \mathrm{a}, \mathrm{c}),(23 \mathrm{a}, \mathrm{c})$, and $(25 \mathrm{a}, \mathrm{b})$ using initial conditions (33). We use the method of lines as described in $\S 3.2$, but now with a uniform mesh in both domains. This reduced model gives excellent agreement with the full problem (12)-(15) (see figure 6), and demonstrates that, in this regime, the important dimensionless parameters are $\mathcal{K}, D_{p}$, and $D_{\phi}$.

4.3. Regime II: Decontamination limited by supply of cleanser. In the regime where $c=0$ at $\xi=1$, the long-time behaviour is limited by the supply of cleanser to the interface. In this regime, the interfacial conditions (24) become

$$
\begin{aligned}
c & =0, \\
\frac{S-1}{S} c_{\xi}-D_{\phi} \phi_{\eta} & =(1-S) S_{T}(1-\phi), \\
D_{p} p_{\xi} & =S S_{T}(1-p), \\
\mathcal{K} p & =\phi .
\end{aligned}
$$

The leading-order system for Regime II is given by (22), (23), and (34). To solve this system numerically, we must also calculate appropriate 'initial' conditions as $T \rightarrow 0^{+}$. Thus, just as we did with Regime I, we now look for a small-time solution to the reduced problem in Regime II, which we again refer to as the intermediate-time solution. The scalings are the same in this regime, and we seek solutions using the formal scalings $T=\delta \tilde{T}, \eta=1-\delta^{1 / 2} \tilde{X}$, and $S=2 \lambda(\delta \tilde{T})^{1 / 2}$, where $\delta \ll 1$ is an arbitrary small parameter, and $\lambda$ is a constant which must be determined as part of the solution. We note that the interfacial position again scales with the square root of time in this intermediate-time solution, a scaling often seen in Stefan-type problems 
14 M. P. DALWADI, D. O'KIELY, T. S. KHALEQUE, S. J. THOMSON, AND C. L. HALL
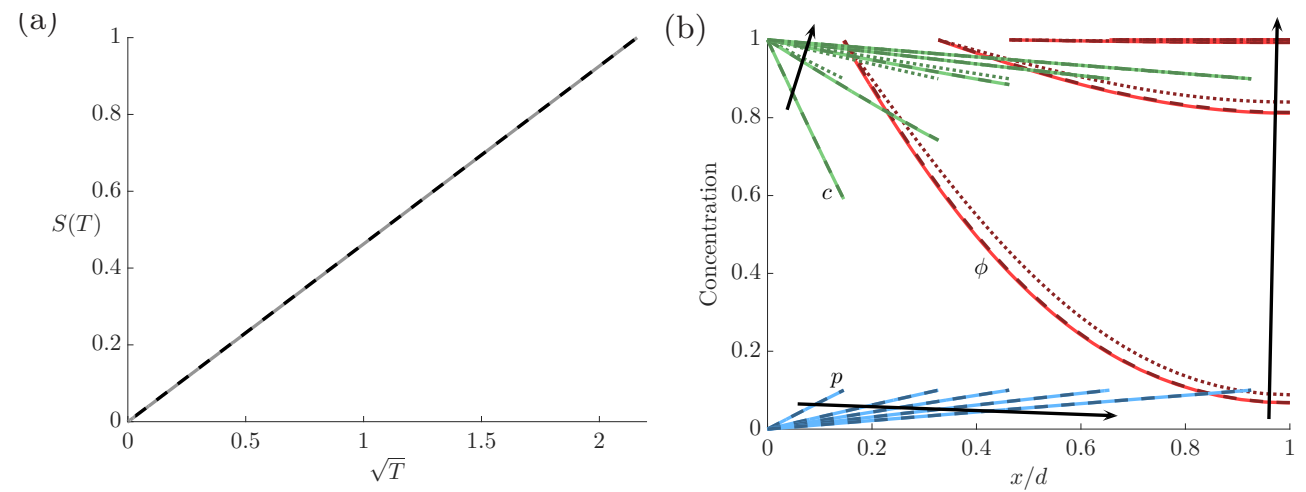

Fig. 6. (Colour online) Comparison of numerical solutions to the full problem (12)-(15) (solid lines) and the large d problem (22a,c), (23a,c), (25a,b), (26) and (27) (dashed lines) in Regime I, for $\beta=D_{\phi}=D_{p}=1, \mathcal{K}=10$, and $d=10^{3}$. (a) The position of the moving boundary. (b) The concentrations of the cleanser, $c$ (green), product in aqueous phase, $p$ (blue), and product in oily phase, $\phi$ (red), at (non-uniform) times $t=1 \times 10^{5}, 5 \times 10^{5}, 1 \times 10^{6}, 2 \times 10^{6}, 4 \times 10^{6}$, with arrows denoting increasing time. The dotted lines show asymptotic solutions $c$ (green dotted) and $\phi$ (red dotted) in the large $\mathcal{K}$ limit, defined in (48) and (45), respectively.

$[3,7]$. However, we shall see that the square-root scaling does not hold throughout this regime.

We look for solutions where $c=c(\xi), p=p(\xi)$, and $\phi=\phi(\tilde{X}, \tilde{T})$ in the system (22), (23), and (34). At leading order in $\delta$, the governing equations (22) are

$$
\begin{aligned}
c_{\xi \xi}+2 \lambda^{2} \xi c_{\xi} & =0, \\
D_{p} p_{\xi \xi}+2 \lambda^{2} \xi p_{\xi} & =0,
\end{aligned}
$$

for $\xi \in(0,1)$, and

$$
D_{\phi} \phi_{\tilde{X} \tilde{X}}+\frac{\lambda}{\tilde{T}^{1 / 2}} \phi_{\tilde{X}}=\phi_{\tilde{T}}
$$

for $\tilde{X}>0$ and $\tilde{T}>0$; the fixed boundary conditions (23) are

$$
c(0)=\beta, \quad p(0)=0 ;
$$

and the interfacial conditions (34) are

$$
\begin{aligned}
c(1) & =0, \\
2 \lambda \tilde{T}^{1 / 2} D_{\phi} \phi_{\tilde{X}}(0, \tilde{T}) & =c_{\xi}(1)+2 \lambda^{2}(1-\phi(0, \tilde{T})), \\
D_{p} p_{\xi}(1) & =2 \lambda^{2}(1-p(1)), \\
\mathcal{K} p(1) & =\phi(0, \tilde{T}) .
\end{aligned}
$$

Finally, the matching condition for $\phi$ is

$$
\phi \rightarrow 0 \text { as } \tilde{X} \rightarrow \infty .
$$

The system (35)-(38) is solved by

$$
c(\xi)=\beta\left(1-\frac{\operatorname{erf}(\lambda \xi)}{\operatorname{erf} \lambda}\right),
$$


(a)

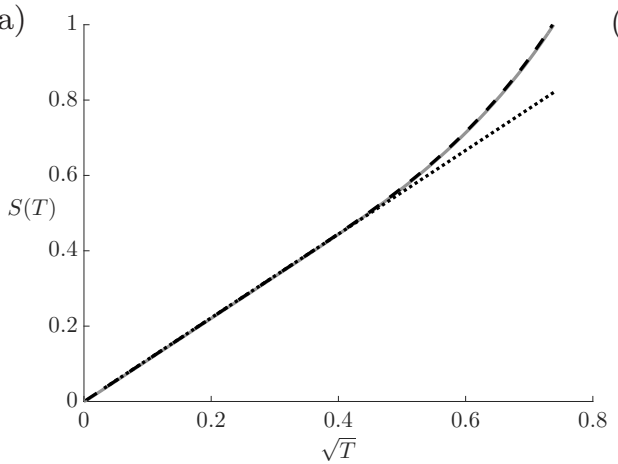

(b)

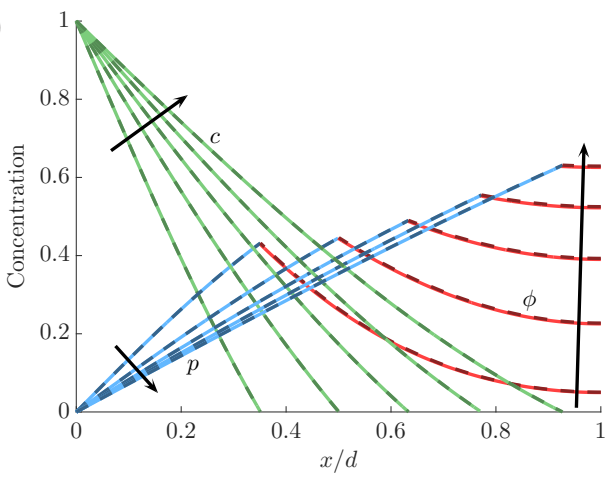

FIG. 7. (Colour online) Comparison of numerical solutions to the full problem (12)-(15) (solid lines) and the large d problem defined by (22), (23), (34), (39), (40) (dashed) in Regime II, for $\beta=D_{\phi}=D_{p}=\mathcal{K}=1$, and $d=10^{3}$. (a) The position of the moving boundary. The dotted black line is $s=2 \lambda t^{1 / 2}$, where $\lambda$ is obtained from the solution to the transcendental equation (40). (b) The concentrations of cleanser, $c$ (green), product in aqueous phase, $p$ (blue), and product in oily phase, $\phi$ (red), at times $t=1 \times 10^{5}, 2 \times 10^{5}, 3 \times 10^{5}, 4 \times 10^{5}, 5 \times 10^{5}$, with arrows denoting increasing time.

$$
\begin{aligned}
p(\xi) & =\frac{\lambda_{p} \operatorname{erf}\left(\lambda_{p} \xi\right)}{\lambda_{p} \operatorname{erf} \lambda_{p}+e^{-\lambda_{p}^{2}} / \sqrt{\pi}}, \\
\phi(\eta, T) & =\frac{\mathcal{K} \lambda_{p} \operatorname{erf} \lambda_{p} \operatorname{erfc}\left((1-\eta) / \sqrt{4 D_{\phi} T}+\lambda_{\phi}\right)}{\left(\lambda_{p} \operatorname{erf} \lambda_{p}+e^{-\lambda_{p}^{2}} / \sqrt{\pi}\right) \operatorname{erfc} \lambda_{\phi}},
\end{aligned}
$$

recalling that $\lambda_{p}=\lambda / D_{p}^{1 / 2}$ and $\lambda_{\phi}=\lambda / D_{\phi}^{1 / 2}$, and where $\lambda$ satisfies the transcendental equation

$$
\lambda_{\phi}-\frac{\beta e^{-\lambda^{2}}}{\sqrt{\pi D_{\phi}} \operatorname{erf} \lambda}=\mathcal{K}\left(\lambda_{\phi}-\frac{e^{-\lambda_{\phi}^{2}}}{\sqrt{\pi} \operatorname{erfc} \lambda_{\phi}}\right) \frac{\lambda_{p} \operatorname{erf}\left(\lambda_{p}\right)}{\lambda_{p} \operatorname{erf} \lambda_{p}+e^{-\lambda_{p}^{2}} / \sqrt{\pi}} .
$$

We note that the long-time solutions to the modified problem with a semi-infinite lower domain in Regime II are given by (39) with $S=2 \lambda T^{1 / 2}$, where $\lambda$ is the solution to $(40)$.

Thus, in Regime II, we have reduced the task of fully understanding our system to numerically solving the system (22), (23), and (34) using initial conditions (39)(40). We use the method of lines with a uniform mesh in both domains. We see that these numerical solutions to the reduced problem (dashed lines) show superb agreement with the solutions to the full problem (solid lines) in figure 7. Moreover, the intermediate-time square root solution to the interfacial position (dotted line in figure 7a) also provides excellent agreement until the lower boundary starts to affect the system.

Although we have derived reduced systems for Regimes I and II in $\S 4.2$ and $\S 4.3$, it is not yet apparent which regime holds for a given set of parameter values. In the next section, we use the results we have derived from our reduced systems to a priori classify the two regimes analytically, in terms of the system parameters.

4.4. Classifying the long-time regime from parameter values. As described in $\S 4.2$ and $\S 4.3$, we find that the position of the moving interface can be approximated by $S=2 \lambda T^{1 / 2}$ throughout Regime I and for early time in Regime II. 


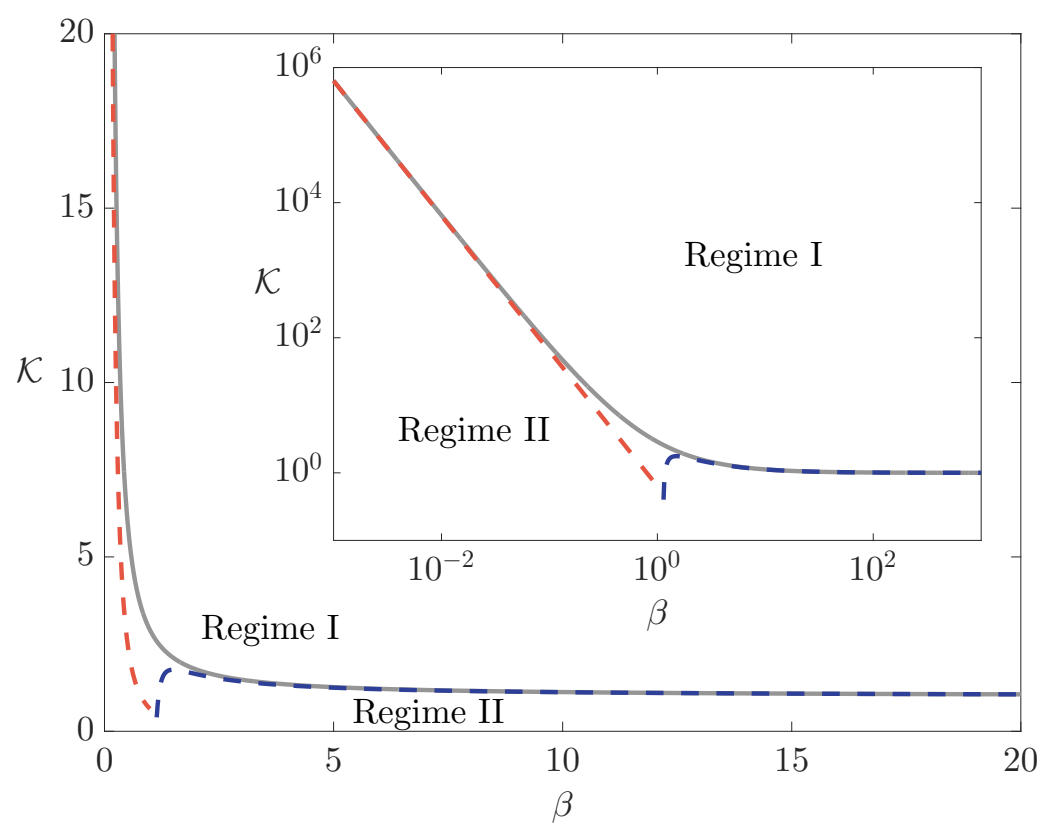

FIG. 8. (Colour online) The critical line described by (27) and (41) in a linear (main) and a log-log plot (inset). The solid grey lines are the numerically determined critical lines, the dashed red lines are from the small $\beta$ /large $\mathcal{K}$ asymptotics (42a), and the dashed blue lines are from the large $\beta /$ small $(\mathcal{K}-1)$ asymptotics given in $(42 \mathrm{~b})$. We use $D_{\phi}=D_{p}=1$ for both figures.

In each case, $\lambda$ is the solution to a transcendental equation, given by (27) for Regime I and (40) for Regime II. The critical line in parameter space associated with the boundary between Regime I and Regime II occurs when both transcendental equations are satisfied. Thus, re-writing (40) using (27) for simplicity, the critical line occurs when

$$
\frac{e^{-\lambda_{p}^{2}}}{\lambda_{p} \sqrt{\pi} \operatorname{erf} \lambda_{p}}=\mathcal{K}-1 \text { and } \frac{\beta e^{-\lambda^{2}}}{\sqrt{D_{\phi}} \operatorname{erf} \lambda}=\frac{e^{-\lambda_{\phi}^{2}}}{\operatorname{erfc} \lambda_{\phi}}
$$

are both satisfied, recalling that $\lambda_{p}=\lambda / D_{p}^{1 / 2}$ and $\lambda_{\phi}=\lambda / D_{\phi}^{1 / 2}$. This critical line may be obtained numerically using a standard root-finding method and we note that the critical line exists for all positive values of $\beta, D_{\phi}$, and $D_{p}$, but is only defined for $\mathcal{K}>1$. In $(\beta, \mathcal{K})$-parameter space, $\mathcal{K} \rightarrow \infty$ as $\beta \rightarrow 0$, and $\mathcal{K}$ monotonically decreases as $\beta$ increases, with $\mathcal{K} \rightarrow 1^{+}$as $\beta \rightarrow \infty$ (figure 8 ). Regime I occurs above the critical line in figure 8 , and Regime II occurs below.

Finally, in the asymptotic limits of small and large $\beta$, we may simplify (41) to obtain

$$
\begin{aligned}
\mathcal{K} & =\frac{2 D_{p} D_{\phi}}{\pi \beta^{2}}+O\left(\beta^{-1}\right) \quad \text { for } \beta \ll 1, \\
\mathcal{K} & \sim 1+\frac{\left(D_{p}(\pi \log \beta)^{1 / D_{p}-1}\right)^{1 / 2}}{\beta^{1 / D_{p}}}\left(1+\frac{\log (\pi \log \beta)}{4 \log \beta}\right) \quad \text { for } \beta \gg 1,
\end{aligned}
$$

and we see that these asymptotic approximations show excellent agreement with the numerical solutions to (41) in their respective limits (figure 8). 
Now that we have classified each regime based on the system parameter values, we can explain when and why the final and effective removal times diverge. As $\mathcal{K}$ increases, more of the product created at the interface goes into the lower oily phase compared to the upper aqueous phase. This dilutes the oily phase near the interface, creating a larger concentration gradient in the oily phase which pushes more agent towards the interface. Thus, significantly more agent is consumed at the interface, so that the proportion of agent remaining in the system drops close to zero before the interface is near the lower boundary.

For a given $\beta$, we can now associate Regime I with larger $\mathcal{K}$, and Regime II with smaller $\mathcal{K}$. We now present some asymptotic results for large and small $\mathcal{K}$.

4.4.1. Large $\mathcal{K}$ results for Regime $I$. We now use asymptotic methods to approximate $t_{f}$ and $t_{e}$ in the limit of large $\mathcal{K}$ in Regime I. In this limit, we can solve (27) to obtain an asymptotic result for $\lambda$, the coefficient governing the interfacial velocity, as follows

$$
\lambda=\sqrt{\frac{D_{p}}{2 \mathcal{K}}}\left(1+\frac{1}{3 \mathcal{K}}+O\left(\mathcal{K}^{-2}\right)\right) \quad \text { as } \mathcal{K} \rightarrow \infty,
$$

and thus we see that the interfacial velocity is slower when $\mathcal{K}$ is large, and the leadingorder velocity is inversely proportional to the square root of $\mathcal{K}$. Combining (28) and (43), we further deduce that

$$
\frac{t_{f}}{d^{2}} \sim \frac{1}{2 D_{p}}\left(\mathcal{K}-\frac{2}{3}\right) \quad \text { for } \mathcal{K} \rightarrow \infty, d \rightarrow \infty,
$$

and thus we see that the time taken for the interface to reach the lower boundary scales with $\mathcal{K}$ in this limit. ${ }^{1}$ The large $\mathcal{K}$ asymptotic results for $t_{f}$ in figure 9 (green line) show excellent agreement with the numerical solutions (black addition signs), even for lower values of $\mathcal{K}$.

Moreover, using the slow interfacial velocity result, we can also obtain an asymptotic solution for $\phi$ when $\mathcal{K} \rightarrow \infty$, and hence for $t_{e}$. In this limit, the leading-order equation for $\phi$ becomes

$$
D_{\phi} \phi_{\eta \eta}=\left(2 \lambda T^{1 / 2}-1\right)^{2} \phi_{T}
$$

valid when $0<T<1 /\left(4 \lambda^{2}\right)$, noting that $\lambda$ is given by (43), and subject to the initial and boundary conditions

$$
\phi(\eta, 0)=0 \quad \phi_{\eta}(0, T)=0 \quad \phi(1, T)=1 .
$$

The system (45) is solved by

$$
\phi=1-\sum_{n=0}^{\infty} \frac{2(-1)^{n}}{w_{n}} \exp \left\{-\frac{w_{n}^{2} D_{\phi}}{2 \lambda^{2}}\left[\frac{2 \lambda T^{1 / 2}}{1-2 \lambda T^{1 / 2}}+\log \left(1-2 \lambda T^{1 / 2}\right)\right]\right\} \cos w_{n} \eta
$$

where $w_{n}=\pi(2 n+1) / 2 .^{2}$ We see that (46), the asymptotic solution for $\phi$, shows good agreement with the full numerical solution (figure 6).

\footnotetext{
${ }^{1}$ We could also have obtained (43) and (44) by directly considering the limit $\mathcal{K} \rightarrow \infty$ in (25).

${ }^{2}$ We note that the system (45) and solution (46) could also have been derived by investigating the asymptotic region where $T=O\left(1 / \lambda^{2}\right)$ and looking for a solution of the form $\phi=$ $1+\exp \left(-g\left(\lambda^{2} T\right) / \lambda^{2}\right) f(\eta)$.
} 


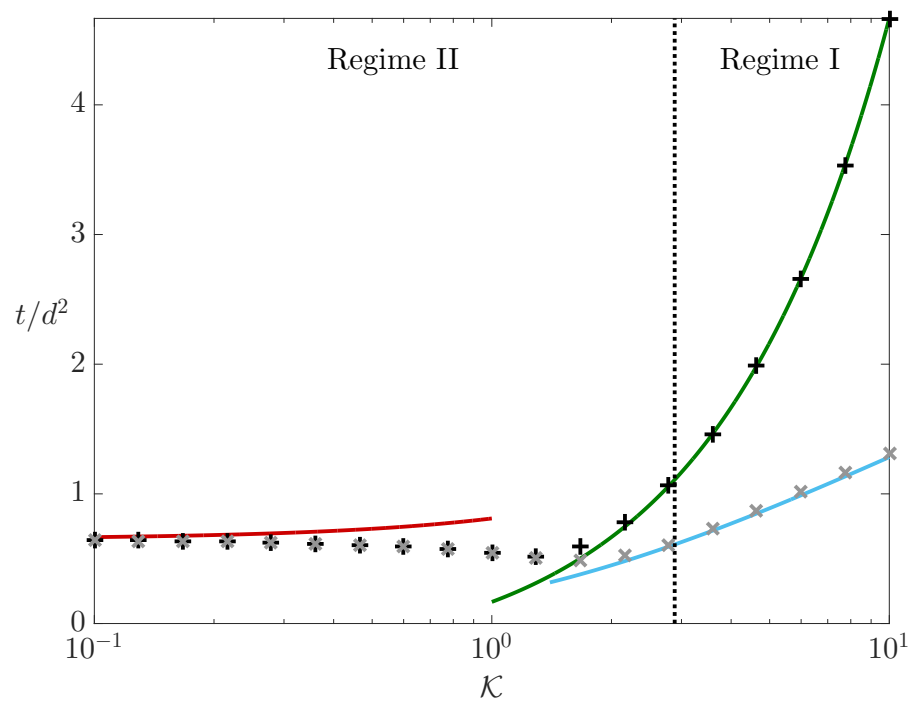

FIG. 9. (Colour online) The scaled final time $t_{f} / d^{2}$ and effective removal time $t_{e} / d^{2}$ as functions of $\mathcal{K}$ for $\beta=D_{\phi}=D_{p}=1$ and $d=10^{3}$. The black addition signs are the numerically determined final times and the grey multiplication signs are the numerically determined effective removal times, both from the full problem defined in (12)-(15). For large $\mathcal{K}$, we plot asymptotic approximations of $t_{f} / d^{2}$ (green) and $t_{e} / d^{2}$ (blue), from (44) and (47), respectively. For small $\mathcal{K}$, the final and effective removal times coincide, and we approximate both with the red line using (28). The vertical black dotted line at $\mathcal{K}=2.885$ denotes the position of the critical line between regimes, defined by (41).

The asymptotic solution (46) allows us to approximate $t_{e}$. From (20c) and (46), we see that $t_{e}$ can be approximated by $t_{e} \sim d^{2} T^{*}$, where $T^{*}$ is a solution to $F\left(T^{*}\right)=\varepsilon$ and $F(T)$ is defined by

$$
F(T)=\left(1-2 \lambda T^{1 / 2}\right) \sum_{n=0}^{\infty} \frac{2}{w_{n}^{2}} \exp \left\{-\frac{w_{n}^{2} D_{\phi}}{2 \lambda^{2}}\left[\frac{2 \lambda T^{1 / 2}}{1-2 \lambda T^{1 / 2}}+\log \left(1-2 \lambda T^{1 / 2}\right)\right]\right\} .
$$

Furthermore, as $\lambda=O(1 / \sqrt{\mathcal{K}})$ is small in this limit, we can approximate $F(T)$ by the first term of the infinite sum in (47). Making all of these simplifications, estimating $t_{e}$ reduces to the problem of numerically solving a transcendental equation. We see excellent agreement between the asymptotic approximation of $t_{e}$ (blue line) and the full numerical results (grey multiplication signs), again even for only moderately large values of $\mathcal{K}$ (figure 9 ).

We note that the interfacial condition (25b) is greatly simplified in this limit, if $\phi \approx 1$. In this scenario $c=c(\xi)$, and

$$
c(\xi)=\beta\left(1-\frac{\lambda \operatorname{erf} \lambda \xi}{\lambda \operatorname{erf} \lambda+e^{-\lambda^{2}} / \sqrt{\pi}}\right),
$$

towards the end of the decontamination in Regime I. In figure 6, we confirm that (48) only shows good agreement with the full numerical solution when $\phi \approx 1$.

Finally, our large $\mathcal{K}$ analysis shows that $t_{e}$ and $t_{f}$ are independent of $\beta$ in this regime. This is because the cleanser dynamics are not important in this regime; the important mechanism is product removal from the oily phase. In general, a large $D_{\phi}$ 
results in quicker transport of agent to the interface, with the effect of decreasing $t_{e}$, the effective removal time, and a large $D_{p}$ results in quicker transport of product in the aqueous phase to the upper boundary, with the effect of decreasing $t_{f}$, the final time. We note that there is another distinguished asymptotic limit of this system when $D_{p}$ is as large as $d$, but the analysis of this limit is beyond the scope of this paper.

4.4.2. Small $\mathcal{K}$ results for Regime II. In contrast to Regime I, the final time and effective removal times almost coincide in Regime II (figures 5 and 9). Naively, one might hypothesise that the interfacial velocity $S=2 \lambda T^{1 / 2}$, with $\lambda$ defined in (40) for the intermediate-time system, would be a good approximation of the interfacial velocity throughout the process. However, our numerical solutions show that this only gives a good estimate for the removal time in the limit $\mathcal{K} \rightarrow 0$ (red line in figure 9); the discrepancy between (39c) and (23c), the boundary condition at $\eta=0$, when $1-\eta=O\left(T^{\frac{1}{2}}\right)$ is not small means that the intermediate-time system cannot generally be used to estimate removal time.

In light of this, and having briefly discussed the limit $\mathcal{K} \rightarrow 0$ at the end of $\S 2.2$, we now consider this limit in more detail. Our aim is to explain the accuracy of the removal time naively estimated by the intermediate-time solution when $\mathcal{K} \rightarrow 0$. In this limit, we find that $\phi=O(\mathcal{K})$, and hence the long-time interfacial conditions (24) become, to leading order in $\mathcal{K}$,

$$
\begin{aligned}
c & =0, \\
c_{\xi}+S S_{T} & =0, \\
D_{p} p_{\xi} & =S S_{T}(1-p), \\
\mathcal{K} p & =\phi,
\end{aligned}
$$

for $\xi, \eta=1$ with $T>0$. Hence, the system decouples for $c$ and $S$, which are now governed by the classical Stefan problem with interfacial conditions $(49 a, b)$; in this simplified system, $\beta$ acts as the inverse Stefan number [3, 7]. Thus, the solutions for $c$ and $p$ in this limit are the same as for the intermediate-time problem, given by (39a) and (39b), with $S=2 \lambda T^{1 / 2}$, where $\lambda$ satisfies the transcendental equation

$$
\lambda=\frac{\beta \exp \left(-\lambda^{2}\right)}{\sqrt{\pi} \operatorname{erf} \lambda} .
$$

Although $\phi$ must be solved using the full equation (22c) with the interfacial condition (49d), the small $\mathcal{K}$ result for the interfacial velocity over the long timescale, which we present above, agrees with the intermediate-time result for the interfacial velocity for small $\mathcal{K}$, presented in $\S 4.3$. This can be seen from the agreement between the small $\mathcal{K}$ limit in (40) and (50). Thus, we have shown that the intermediate-time interfacial velocity becomes valid for all time in the limit of small $\mathcal{K}$, explaining why the naive intermediate-time result for the removal time becomes accurate in the same limit.

\section{Discussion and conclusions.}

5.1. Dimensional results. The asymptotic results in $\S 4$ are all obtained by considering a 'deep' spill of agent; that is, we assume throughout that $d:=\bar{L} \bar{k} / \bar{D}_{c}$ is large. We find that this leads to two different regimes, one where the rate of decontamination is limited by the removal of product from the system (Regime I, described in $\S 4.2$ ), and another where the rate of decontamination is limited by the supply of cleanser to the interface between the phases (Regime II, described in §4.3). 
Our asymptotic results are stated in terms of the dimensionless model developed in $\S 2.3$, but it is also valuable to examine them in dimensional form. We find that the leading order results for the dimensional removal time often depend on only some of the eight dimensional parameters introduced in $\S 2.1$ and $\S 2.2\left(\bar{L}, \bar{D}_{c}, \bar{D}_{p}, \bar{D}_{\phi}\right.$, $\bar{c}_{0}, \bar{V}_{m}, \bar{k}$, and $\left.\mathcal{K}\right)$. Information about which dimensional parameters appear in the leading order expression for the removal time has the potential to be particularly valuable to experimental researchers, since it indicates the parameters that have the most influence on the time taken to remove a harmful agent.

In Regime I, reversing the nondimensionalisation of (28) gives the result that the dimensional time to complete removal, $\bar{t}_{f}[\mathrm{~s}$ ], is given to leading order by

$$
\bar{t}_{f} \sim \frac{\bar{L}^{2}}{4 \bar{D}_{p} \lambda_{p}(\mathcal{K})}
$$

where $\lambda_{p}(\mathcal{K})$ is implicitly defined by $(27)$. Hence, if $d=\bar{L} \bar{k} / \bar{D}_{c}$ is large and we satisfy the conditions in $\S 4.4$ in order to be in Regime I, the leading order time to complete removal depends only on $\bar{L}, \bar{D}_{p}$, and $\mathcal{K}$.

Following the analysis that leads to (44), we further simplify this to obtain

$$
\bar{t}_{f} \sim \frac{\bar{L}^{2}}{2 \bar{D}_{p}} \mathcal{K}-\frac{\bar{L}^{2}}{3 \bar{D}_{p}}
$$

as long as $\mathcal{K}$ and $\bar{L} \bar{k} / \bar{D}_{c}$ are both large.

In Regime I, we recall from figures 5 and 9 that the effective removal time, $t_{e}$, may be very different from the final time, $t_{f}$, and that $t_{e}$ may be a better measure of the decontamination time than $t_{f}$. Starting from (47) and reversing the nondimensionalisation, we find that the dimensional effective removal time, $\bar{t}_{e}[\mathrm{~s}]$, is given to leading order in large $\bar{L} \bar{k} / \bar{D}_{c}$ and large $\mathcal{K}$ by

$$
\bar{t}_{e} \sim \frac{\bar{L}^{2}}{4 \bar{D}_{p} \lambda_{p}(\mathcal{K})} \tau\left[\frac{\bar{D}_{\phi}}{\bar{D}_{p} \lambda_{p}(\mathcal{K})^{2}}\right],
$$

where $\tau(R)$ is implicitly defined by

$$
\varepsilon=\frac{8\left(1-\tau^{\frac{1}{2}}\right)}{\pi^{2}} \exp \left\{-\frac{\pi^{2} R}{8}\left[\frac{\tau^{1 / 2}}{1-\tau^{1 / 2}}+\log \left(1-\tau^{1 / 2}\right)\right]\right\},
$$

where $\varepsilon$ is the threshold introduced in (20c), and $\lambda_{p}(\mathcal{K})$ is implicitly defined by (27) as before. The dimensional effective removal time is therefore (to leading order) a function of $\bar{L}, \bar{D}_{p}, \bar{D}_{\phi}$ and $\mathcal{K}$ only. This further illustrates the fact that, in Regime I, the removal of the agent is relatively insensitive to the cleanser dynamics. In particular, increasing the cleanser concentration, $\bar{c}_{0}$, or using a cleanser with a higher diffusivity, $\bar{D}_{c}$, has very little effect on the agent removal time, provided the parameters already satisfy the conditions to be in Regime I.

In Regime II, we expect the cleanser dynamics to be more important, since the rate-limiting process is the supply of cleanser to the interface between the phases. While Regime II is more complicated than Regime I, we again find that the leadingorder dimensional removal time is independent of some model parameters. We recall that we are able to obtain an 'intermediate time' solution in Regime II because (after an initial transient) the solution is insensitive to the initial conditions imposed. By 
inspection of (22), (23), and (34), we note that the intermediate time problem is independent of $d=\bar{L} \bar{k} / \bar{D}_{c}$. Hence, by reversing the nondimensionalisation and applying the long time scaling from (21), we see that the dimensional removal time must take the form

$$
\bar{t}_{f} \sim \frac{\bar{L}^{2}}{\bar{D}_{c}} f\left[\bar{c}_{0} \bar{V}_{m}, \frac{\bar{D}_{p}}{\bar{D}_{c}}, \frac{\bar{D}_{\phi}}{\bar{D}_{c}}, \mathcal{K}\right]
$$

where $f$ is some function. Thus, $\bar{t}_{f}$ must be independent of $\bar{k}$ to leading order; even in Regime II, changing the reaction rate constant has only a small effect on the removal time. Furthermore, when $\mathcal{K}$ is small, we find that

$$
\bar{t}_{f} \sim \frac{\bar{L}^{2}}{4 \bar{D}_{c} \lambda\left(\bar{c}_{0} \bar{V}_{m}\right)},
$$

where $\lambda(\beta)$ is defined implicitly by (50). Hence, for sufficiently small $\mathcal{K}$ we find that, to leading order, $\bar{t}_{f}$ depends only on $\bar{L}, \bar{D}_{c}, \bar{c}_{0}$ and $\bar{V}_{m}$; apart from the dependence on $\bar{V}_{m}$, the removal time is completely independent of the properties of the agent and the reaction product.

5.2. The desirable features of a cleanser. In many cases, a range of different cleanser solutions could be used against the same agent [14, 19]. Choosing an appropriate cleanser depends on a number of factors (for example, the possibility of chemical reactions between the cleanser and the substrate), but one important factor is the speed with which the cleanser will eliminate an agent. This has been the focus of our analysis, our results can be used to indicate how properties such as the cleanser concentration, the cleanser potency (as measured by the effective rate constant), and the cleanser reaction mechanism affect the speed of decontamination. Some cleanser properties that one might expect to be important turn out to have only a minor effect on the speed of decontamination. This insight is valuable in highlighting how to focus efforts and resources when choosing a cleanser for a given task.

We find that the leading-order dimensional removal time does not depend on the cleanser concentration applied at the surface, $\bar{c}_{0}$, in Regime I, but does depend on $\bar{c}_{0}$ in Regime II. If the reaction product is more soluble in water than oil (and hence $\mathcal{K}<1$ ), the relevant parameter regime will always be Regime II and increasing $\bar{c}_{0}$ will always decrease the removal time (albeit with diminishing returns). Moreover, when $\mathcal{K} \rightarrow 0$ we can use (50) and (56) to obtain asymptotic results for the removal time as a function of $\bar{c}_{0}$.

If the reaction product is more soluble in oil than water (and hence $\mathcal{K}>1$ ), we observe from figure 8 that increasing $\bar{c}_{0}$ (and hence $\beta$ ) will lead to a transition from Regime II to Regime I. Hence, increasing the cleanser concentration will only cause significant decreases in removal time up to the point where removal of reaction product (and hence availability of agent at the reaction interface) supersedes cleanser availability as the rate-limiting step of decontamination. Thereafter, further increases in cleanser concentration will not lead to significant improvements in decontamination speed.

Our analysis also shows that the leading-order removal time is independent of the effective rate constant $\bar{k}$ in both Regime I and Regime II. If $\bar{k}$ is sufficiently small that the 'deep spill of agent' assumption is no longer valid, then the decontamination behaviour may change significantly. However, as long as $\bar{k} \gg \bar{D}_{c} / \bar{L}$, changing the reactivity of a cleanser will only yield a small change in removal time. This suggests 
TABLE 1

Reaction products and typical parameters for three cleansers that can be used to neutralise sulfur mustard. We estimate the molar volume of sulfur mustard $\bar{V}_{m}=1.2 \times 10^{-3} \mathrm{~m}^{3} \mathrm{~mol}^{-1}$ by combining density [14] and molar mass information.

\begin{tabular}{r|llll} 
Cleanser & DS2 & $5 \%$ Bleach sol. & $\mathrm{Ca}(\mathrm{OH})_{2}$ sol. & Ref. \\
\hline Product & Divinyl sulfide & Mustard sulfoxide & Thiodiglycol & {$[14,17]$} \\
$\bar{c}_{0}\left(\mathrm{~mol} \mathrm{~m}^{-3}\right)$ & $6.7 \times 10^{3}$ & $6.7 \times 10^{2}$ & $2.5 \times 10^{1}$ & {$[14,17]$} \\
$\beta=\bar{V}_{m} \bar{c}_{0}$ & 8 & $8 \times 10^{-1}$ & $3 \times 10^{-2}$ & \\
$\mathcal{K}$ & 7.1 & $1.4 \times 10^{-1}$ & $1.7 \times 10^{-1}$ & {$[14]$}
\end{tabular}

that replacing an effective cleanser with an even more potent cleanser (where the reaction products are the same but the reaction is faster) will not significantly improve decontamination speed.

In contrast, the decontamination time depends strongly on the partition coefficient of the reaction product, $\mathcal{K}$. If all other parameters are kept fixed, we find that there is an optimal value of $\mathcal{K}$ for which the removal time is minimised, as illustrated in figures 5 and 9 . However, since there are typically only two or three reaction pathways that can be used to neutralise a given agent $[14,21]$, there is only limited scope for tuning $\mathcal{K}$ to be close to the optimal value.

That said, our analysis shows that the removal time increases linearly with large $\mathcal{K}$, while it approaches a constant as $\mathcal{K} \rightarrow 0$. This suggests that, as a rule of thumb, a cleanser that leads to a reaction product that is exclusively (or almost exclusively) soluble in the water phase will lead to faster decontamination than a cleanser that leads to a reaction product that is exclusively (or almost exclusively) soluble in the oil phase. While an intermediate value of $\mathcal{K}$ may lead to still faster decontamination, we expect that small $\mathcal{K}$ will be preferable to large $\mathcal{K}$ in many situations.

5.3. Decontamination of sulfur mustard. We now consider a specific example, the decontamination of sulfur mustard. Three examples of cleansers that could be used to neutralise sulfur mustard are Decontamination Solution 2 (DS2), a 5\% bleach solution, or a saturated calcium hydroxide solution $[14,17]$. In each case, the mechanism of decontamination is different, leading to different reaction products.

From experimental results for each cleanser, we can estimate the concentrations of active ingredient $\left(\bar{c}_{0}\right)$, the key product formed, and estimated oil-water partition coefficients $(\mathcal{K})$ based on octanol-water partition coefficients given in [14], and we state these values in table 1 . However, it is more difficult to obtain data on relevant diffusivities and effective reaction coefficients. It should also be noted that the mechanisms of decontamination of sulfur mustard are far more complicated than the simple bimolecular reaction we propose in this paper; despite this, we hope to gain valuable insights into the dominant kinetics of decontamination using our model analysis.

For each of the three cleansers, we determine whether the decontamination reaction takes place in Regime I or Regime II, making the assumption $D_{p}=D_{\phi}=1$. We find that the reaction is in Regime II for each cleanser, so that increasing cleanser concentration speeds up decontamination. With DS2, however, the decontamination reaction will be close to the boundary between Regime I and Regime II, and it is possible that increases in cleanser concentration will be less effective. Since diethylenetriamine, the active ingredient in DS2, is highly reactive and corrosive, this might even suggest that reducing the concentration of diethylenetriamine in DS2, and hence increasing the amount of time that it could be applied before the substrate becomes 
damaged, might be an effective strategy for improving the efficiency of decontamination.

5.4. Conclusions. In this paper, we have presented and analysed a model of chemical decontamination that reveals how different features of a cleanser affect the speed of decontamination. We consider a one-dimensional porous medium of finite depth, fully saturated with a chemical agent. Initially, a cleanser in aqueous solution is applied at the top of the porous medium. To the best of our knowledge, this together with a study group report on preliminary work [6] are the first models of reactive decontamination where the reacting species are in different fluid phases; our model could therefore form the foundation for a range of future modelling work on chemical decontamination and similar processes. We note that, since the medium is fully saturated and the porous medium is inert, the system under consideration is a diffusion problem with a reaction at the moving interface between the two fluid phases. Future extensions of this work might include the effects of advection within each fluid phase, using Darcy's law. One could model the effect of scrubbing at the surface by pressure-driven forcing of the cleanser solution through the porous medium, and scenarios where the medium is only partially saturated, in which the fluid dynamics could be modelled using Richards' equation.

The problem under consideration here is a moving-boundary problem with some similarities to the classical Stefan problem with kinetic undercooling, but we find that the precise behaviour is markedly different in different parameter regimes. In the limit where the initial agent layer is deep compared to diffusive lengthscales, we identify two distinct parameter regimes in which the rate of decontamination is limited by either the transport of cleanser or the transport of reaction product. In each case we determine the long-time behaviour and hence removal time in this asymptotic limit. Our asymptotic analysis shows that, to leading order, the time required to remove the agent only depends on some of the model parameters. Importantly, we find that the removal time is independent of the effective rate constant in all parameter regimes considered here. This indicates that using a more potent cleanser (one where the rate of reaction between cleanser and agent is faster) may not lead to significant improvements in removal time. Moreover, we find that changing cleanser concentration only affects the removal time in certain parameter regimes. In fact, the oil-water partition coefficient of the reaction product appears to be more significant in determining the time taken the remove the agent; for given values of the remaining system parameters, this partition coefficient has an optimal value that minimises the removal time.

The work in this paper was motivated by the extreme difficulty of performing experiments using live agents, due to safety and visualisation challenges. By contrast, mathematical modelling allows for the exploration of many hypothetical scenarios. It is our hope that the model and analysis presented in this paper will guide the development and improvement of methods used by the chemical decontamination community, and provide inspiration for further study of this topic.

Acknowledgments. This work originated from a problem presented by the UK Government Decontamination Service (UKGDS) at the 100th European Study Group with Industry (ESGI100), held at the Mathematical Institute, University of Oxford in April 2014. The original ESGI100 report can be accessed at http://www.maths-inindustry.org $/ \mathrm{miis} / 671 /$. The authors would like to acknowledge the valuable contribution made by scientists from the UKGDS, especially Dr Hasmitta Stewart and Mr Tony Arkell. CLH also acknowledges support from the UK Home Office through the Small Business Research Initiative for 'Chemical Decontamination' and the support of 


\section{M. P. DALWADI, D. O'KIELY, T. S. KHALEQUE, S. J. THOMSON, AND C. L. HALL}

the Mathematics Applications Consortium for Science and Industry (www.macsi.ul.ie) funded by the Science Foundation Ireland grant investigator award 12/IA/1683. Finally, the authors would like to thank the referees for their helpful comments.

\section{REFERENCES}

[1] C. M. Boone, Present state of CBRN decontamination methodologies, tech. report, TNO Defence, Security and Safety, 2007.

[2] H. S. Carslaw and J. C. Jaeger, Conduction of heat in solids, Oxford: Clarendon Press, 1959, 2nd ed., 1959.

[3] J. Crank, Free and moving boundary problems, Clarendon Press Oxford, 1984.

[4] V. Cvetkovic and G. Dagan, Reactive transport and immiscible flow in geological media. ii. applications, in Proc. Roy. Soc. Lond. A Math., vol. 452, The Royal Society, 1996, pp. 303-328.

[5] G. Dagan and V. Cvetkovic, Reactive transport and immiscible flow in geological media. i. general theory, in Proc. Roy. Soc. Lond. A Math., vol. 452, The Royal Society, 1996, pp. 285-301.

[6] M. Dalwadi, E. Dubrovina, A. Eisenträger, A. Lee, J. Maestri, B. Matejczyk, D. O'Kiely, M. Stamper, And S. Thomson, Toxic chemicals and their neutralisation agents in porous media, tech. report, ESGI100 (http://www.maths-inindustry.org/miis/671/), 2014.

[7] S. H. DAvis, Theory of solidification, Cambridge University Press, 2001.

[8] J. D. Evans And J. KIng, Asymptotic results for the Stefan problem with kinetic undercooling, Q. J. Mech. Appl Math., 53 (2000), pp. 449-473.

[9] A. A. Fattah, J. A. Barrett, R. D. J. Arcilesi, K. J. Ewing, C. H. Lattin, M. S. HelinSKI, AND I. A. BAIG, Guide for the selection of chemical and biological decontamination equipment for emergency first responders, tech. report, NIJ Guide 103-00, 2001.

[10] K. Kim, O. G. Tsay, D. A. Atwood, And D. G. Churchill, Destruction and detection of chemical warfare agents, Chem. Rev., 111 (2011), pp. 5345-5403.

[11] K. V. Kumar, K. PORKOd, AND F. ROCHA, Langmuir-Hinshelwood kinetics: A theoretical study, Catalysis Communications, 9 (2008), pp. 82-84, doi:http://dx.doi.org/10.1016/j.catcom.2007.05.019.

[12] P. C. Lichtner, Continuum model for simultaneous chemical reactions and mass transport in hydrothermal systems, Geochim. Cosmochim. Acta, 49 (1985), pp. 779-800.

[13] P. C. Lichtner, The quasi-stationary state approximation to coupled mass transport and fluidrock interaction in a porous medium, Geochim. Cosmochim. Acta, 52 (1988), pp. 143-165.

[14] N. B. Munro, S. S. Talmage, G. D. Griffin, L. C. Waters, A. P. Watson, J. F. King, AND V. Hauschild, The sources, fate, and toxicity of chemical warfare agent degradation products, Environmental Health Perspectives, 107 (1999), pp. 933-972.

[15] C. F. Novak, L. W. Lake, And R. S. Schechter, Geochemical modeling of two-phase flow with interphase mass transfer, AIChE journal, 37 (1991), pp. 1625-1633.

[16] E. RABER AND R. MCGuire, Oxidative decontamination of chemical and biological warfare agents using L-Gel, Journal of Hazardous Materials, 93 (2002), pp. 339-352, doi:10.1016/S0304-3894(02)00051-1.

[17] D. H. Rosenblatt, M. J. Small, T. A. Kimmell, and A. W. Anderson, Background chemistry for chemical warfare agents and decontamination processes in support of delisting waste streams at the US Army Dugway Proving ground, Utah, tech. report, Argonne National Lab., IL (United States), 1996.

[18] J. Rubin, Transport of reacting solutes in porous media: Relation between mathematical nature of problem formulation and chemical nature of reactions, Water Resour. Res., 19 (1983), pp. 1231-1252.

[19] B. Singh, P. G. K, K. S. Pandey, R. K. Danikhel, and R. Vijayaraghavan, Decontamination of chemical warfare agents, Defence Science Journal, 60 (2010), pp. 428-441.

[20] A. R. Wilmsmeyer, W. O. Gordon, E. D. Davis, B. A. Mantooth, T. A. Lalain, and J. R. MORRIS, Multifunctional ultra-high vacuum apparatus for studies of the interactions of chemical warfare agents on complex surfaces, Review of Scientific Instruments, 85 (2014), p. 014101.

[21] Y.-C. YANG, J. A. BAKER, AND J. R. WARD, Decontamination of chemical warfare agents, Chemical Reviews, 92 (1992), pp. 1729-1743. 